\title{
CON-FORMACIÓN DE LA MERCANCÍA-BOLAÑO. LA VIDA EDITORIAL de Roberto Bolaño según Jorge Herralde
}

\author{
Alfredo Léal Rodríguez \\ Universidad Nacional Autónoma de México, México
}

\begin{abstract}
Resumen: En su texto "Vida editorial de Roberto Bolaño", publicado a sólo dos años de la muerte del autor, el editor español Jorge Herralde traza las coordenadas para leer la obra de Bolaño a la luz de los diversos procesos editoriales a los que estuvo sometida. El esquema de vida que traza Herralde es objeto de una subsunción real a la forma artificial que él mismo denomina "vida editorial", la cual podríamos entender menos como una mercantificación de la vida que como una antropomorfización de la obra. Desde una perspectiva interdisciplinar que se apoya en la crítica de la cultura de Bolívar Echeverría, el presente artículo trata de mostrar de qué manera esta con-formación de la vida de Bolaño en torno a su vida editorial constituye un primer y decisivo paso para el paradigma de la producción, circulación y consunción de autores en Latinoamérica a inicios del siglo XXI.

Palabras clave: Roberto Bolaño; Jorge Herralde; mercantificación; vida editorial; crítica de la cultura.
\end{abstract}

Recibido: 27 de mayo de 2019

Aprobado: 17 de agosto de 2019

\section{Con-formation of Bolaño-merchandise. The editorial life of Roberto Bolaño according to Jorge Herralde}

\begin{abstract}
In his text "Roberto Bolaño's editorial life", published only two years after the author's death, the Spanish editor Jorge Herralde traces the coordinates to read Bolaño's work in light of the various editorial processes to which he was subjected. The scheme of life that Herralde draws is the object of a real subsumption to the artificial form that he calls "editorial life", which we could understand less as a
\end{abstract}

\footnotetext{
${ }^{1}$ Alfredo Lèal (San Pedro Mártir, 1985). Escritor, traductor y docente. Licenciado y Maestro en Letras Modernas Francesas por la UNAM, donde actualmente es Doctorando en Estudios Latinoamericanos. Ha sido profesor de la Facultad de Filosofía y Letras de la UNAM y de la Universidad Iberoamericana Puebla. Ha participado en numerosos coloquios nacionales e internacionales en los campos de la literatura, la sociología literaria y la historiografía. Asimismo, es el primer traductor mexicano de Marcel Proust al español. Correo electrónico: alfredo.leal. rodriguez@gmail.com
} 
mercantification of life than as an anthropomorphization of the work. From an interdisciplinary perspective based on Bolívar Echeverría's critique of culture, this article aims to show how this con-formation of Bolaño's life around his editorial life constitutes a first and decisive step for the paradigm of the production, circulation and consumption of authors in Latin America at the beginning of the 21st century.

Keywords: Roberto Bolaño; mercatification; literary criticism; cultural criticism.

"Macedonio Fernández no vende". Esa es la frase central de la famosa conferencia que Bolaño impartió en Sevilla, recopilada por Ignacio Echevarría en El secreto del mal e intitulada "Sevilla me mata" (Bolaño 179). ¿Por qué regresar a este enunciado? Porque es en las condiciones de posibilidad del mismo donde, creemos, se encuentra la clave para trazar una genealogía de las prácticas editoriales finiseculares del siglo XX y de inicios del siglo XXI en Latinoamérica. Dicho de otro modo: en esa línea, "Macedonio Fernández no vende”, enunciada por Bolaño en la que para muchos fue su consagración como el escritor más influyente, el "hermano mayor", como se dirá en El País, de la generación de Rodrigo Fresán, Santiago Gamboa y Cristina Rivera $\mathrm{Garza}^{2}$, en esa sola línea se plantea no sólo cuál es el camino a seguir por la Literatura Latinoamericana en el siglo XXI sino, más importante aún, cuál es su procedencia. Hay que regresar, pues, una y otra vez, a nuestro punto de partida: "Macedonio Fernández no vende", pero en esta ocasión hay que oponerle una línea, en apariencia muy simple, pues aun cuando sea él mismo quien enuncie esa línea, "Macedonio Fernández no vende", lo cierto es que Bolaño sí, lo cierto es que Roberto Bolaño sí vende.

\footnotetext{
${ }^{2}$ El artículo, publicado el 29 de enero de 2004 bajo el título "Los herederos del 'boom' defienden una literatura en español sin fronteras", es una nota —en constante referencia a la reunión de Sevilla - sobre la presentación del libro Palabra de América, publicado por Seix Barral en 2004. "El escritor cubano Guillermo Cabrera Infante apoyó con su presencia la reunión y el chileno Roberto Bolaño ofició de 'hermano mayor'. Junto a Bolaño, estuvieron Jorge Franco, Rodrigo Fresán, Santiago Gamboa, Gonzalo Garcés, Fernando Iwasaki, Mario Mendoza, Ignacio Padilla, Edmundo Paz-Soldán, Cristina Rivera Garza, Iván Thays y Jorge Volpi. El libro Palabra de América recoge las intervenciones en las jornadas de estos escritores, que defienden una literatura en español sin fronteras" (Belausteguigoitia).
} 
Dejemos de lado, no obstante, las estadísticas. ${ }^{3}$ La primera pregunta que surge al encontrarnos con estos dos enunciados ("Macedonio Fernández no vende", por un lado; "Roberto Bolaño sí vende", por el otro), más allá de encuadrarlos en una lógica oposicional o dicotómica — es decir, más allá de oponer, por ejemplo, la filiación vanguardista y metafísica de Macedonio (traducida en gran medida por Borges) a las coordenadas realistas e inmanentes en las que se inscribe la obra de Bolaño (deficientemente traducidas en nuestros días por la prosa periodística de la que está saturado el mercado literario)_, e incluso más allá de una posible sociología de la literatura que explicara el contexto en el que surgen ambas obras, la pregunta que me interesa plantear es, creo, mucho más sencilla: ¿por qué sí vende, Bolaño?; ¿por qué es posible decir que "Roberto Bolaño sí vende"? Es decir, se trata de encontrar algo como una sustancia detrás de esta línea (hipotética) que hemos planteado como contraparte o complemento de aquélla enunciada por Bolaño en Sevilla. Se trata, en suma, de una pregunta anterior a aquélla por el estilo o la historia en los que se enmarcan la obra de uno y otro, Macedonio y Bolaño, y plantear una pregunta que se interese por el modo en el que la frase (hipotética) "Roberto Bolaño sí vende" se despliega justamente como instancia nominal en torno a la Literatura Latinoamericana; una pregunta, pues, cuasi arqueológica que trate de dilucidar no la direccionalidad o el telos de esta afirmación sino su sentido, su archè.

\footnotetext{
${ }^{3}$ En el texto " «2666»: datos editoriales", Herralde afirma que "las expectativas respecto a los lectores" de la novela póstuma de Bolaño eran "inciertas" debido a que los libros de Bolaño, "con la excepción de Los detectives salvajes, habían tenido sólo unos pocos miles de lectores" (Herralde Para Roberto Bolaño 49). "Sin embargo", continúa Herralde, "el boca-oreja funcionó arrolladoramente, potenciando el tam tam de la crítica, y las reediciones se han ido sucediendo: la primera, en octubre de 2004, fue de 12,000 ejemplares; la segunda, en noviembre de 2004, de 3,000; la tercera, en diciembre de 2004, de 5,000; la cuarta, en febrero de 2005 de 4,000 y la quinta, en abril de 2005, de 6,000" (Herralde Para Roberto Bolaño 49-50). Un total de 30,000 ejemplares en menos de un año, sólo entre octubre de 2004 y abril de 2005. "En cuanto a la repercusión internacional", continúa Herralde, "[...] en el caso de 2666 también se están superando todas las expectativas en estos tiempos en los que la etiqueta literary fiction despierta un creciente temor, estimulado además por los costos de traducción de un volumen de más de mil páginas. Pese a ello, [para octubre de 2005] ya se [habían] firmado contratos con varios de los editores literarios más prestigiosos del panorama internacional: Adelphi en Italia, Hanser en Alemania y Farrar, Straus \& Giroux en Estados Unidos" (Herralde Para Roberto Bolaño 50). Estos números, que abarcan, quiero insistir en ello, sólo un año - y sólo una novela: 2666 - , pueden servir de indicador y sustento puramente estadístico de las ventas de Bolaño.
} 
Podríamos, acaso, en un primer momento, formular nuestra pregunta de la siguiente manera: puesto que se trata de una instancia nominal y toda instancia nominal implica un sujeto que efectúe la nominación, ¿quién sería capaz de enunciar la frase (hipotética) "Roberto Bolaño sí vende", es decir, quién tendría la capacidad de nombrar a Bolaño en el marco de esta contraposición con respecto a Macedonio? Dicho de otro modo: ¿de dónde podría provenir la frase (hipotética) "Roberto Bolaño sí vende"? En la medida en la que podamos encontrar al agente de dicha discursividad podremos, acaso, entender que esta línea no es sólo el contrario de aquélla con la que hemos empezado a investigar las condiciones de emergencia de la serie de modalidades específicas de subjetivación que vehicula la práctica editorial finisecular y de inicios del siglo XXI en América Latina, sino que se trata de un testimonio del absoluto que se produce en el marco del campo literario si es que el alcance de éste, como creemos, se encuentra delimitado de manera fenomenológica ${ }^{4}$ sobre las líneas de fuga de sus entidades textoautorales. Si se trata, como creemos, de un testimonio del absoluto, de una variante incluida trascendental y fenomenológicamente en la forma de aparecimiento de la propia frase "Macedonio Fernández no vende" —una variante, por decirlo de algún modo, negativa—, esto querría

\footnotetext{
${ }^{4}$ Nos encontramos, como lo habíamos indicado un poco más arriba en esta misma argumentación, con el problema de la delimitación fenomenológica del campo literario - $\mathrm{y}$, decíamos, de cualquier campo, entendido como lo propone la sociología (principalmente de Bourdieu, aunque no precisamente restrictiva a este autor); en palabras de Rancière, "Pour assurer sa royauté, le sociologue a fait bonne mesure. Il a rationnalisé, absolutisé l'arbitraire. À la philosophie, il a opposé une pesanteur de la domination sociale renforcée de la rigueur logique du principe de non-contradiction. Il a doublé la nécessité marxiste par la nécessité parménidienne de son éternité" (Rancière 258). Para entender en profundidad este mecanismo que consiste en absolutizar fenomenológicamente lo arbitrario de la sociedad, es preciso, me parece, que consideremos el testimonio (en cuanto categoría) a la luz de lo que dice Ricœur en su fenomenología del hombre capaz: "Il reste toutefois un écart de sens entre attester et reconnaitre, tenant à leur appartenance à des familles lexicales différentes. Attester appartient à la même famille que le témoignage, lequel se ramifie entre plusieurs acceptions, allant de l'usage du terme dans la conversation ordinaire, à son emploi en historiographie et à la barre du tribunal, et, au-delà, jusqu'à son emploi dans la sphère religieuse, où la valeur du témoignage est attachée, selon l'expression de Jean Nabert, à ces signes contingents que l'absolu donne de lui-même dans l'histoire : on parle alors de «témoins de l'absolu », le témoignage devenant alors le complément existentiel d'une «critériologie du divin » de tour critique comme le mot l'indique" (Ricœur 153). El testimonio entendido desde el campo literario y debido al anclaje estructural que éste tiene en la fenomenología es, para decirlo claramente, una modalidad de ese testimonio del absoluto, con lo cual volvemos a ver las formas de la nominación adánica con las que hemos empezado a analizar el problema de la donación del nombre.
} 
decir que Bolaño no es quien enuncia esa frase. (Y aquí es importante diferenciar entre lo que podemos llamar "Bolaño-autor" y "Bolaño-encuanto-entidad-texto-autoral".) Volvamos, pues, a la conferencia de Sevilla: según lo que cuenta el propio Bolaño, como lo hemos visto anteriormente, alguien le impone el título de la conferencia, alguien le dice que debe decir " $[i]$ De dónde viene la nueva literatura latinoamericana [?]”. ¿Cómo hacer coincidir plenamente una petición ética - a saber, aquélla de determinar el lugar del que procede la nueva literatura latinoamericana - con la donación moral de su propio nombre? En otras palabras, ¿cómo decir que la literatura que constituye las condiciones de posibilidad de la obra de Bolaño y en la que ésta es una coordenada innegable; cómo decir, pues, que la literatura latinoamericana viene de un, digámoslo así, "punto de partida" completamente opuesto al de la realidad que se está desplegando en Sevilla? La única forma de hacer coincidir ambas instancias, a saber, la instancia romántica en la que se reconoce a Macedonio como un producto puramente cultural, "en resistencia deliberada a su aprovechamiento mercantil" (Echeverría 30), a la instancia civilizatoria que atestigua la obra de Bolaño, es decir aquella en la que "la creatividad se ha subordinado al pragmatismo económico" (Echeverría 30) es si las herramientas con las que contamos para el estudio de la literatura latinoamericana se (auto)de-limitan a las propuestas por una idea romántico-germana de la cultura, es decir, si (auto)de-limitamos nuestra actividad a las posibilidades que nos ofrecen la filología o la hermenéutica ${ }^{5}$. No es sólo, entonces, la trascendencia fenomenológica del campo literario sino la aplicación endogámica de la filología y la hermenéutica en cuanto herramientas culturales romántico-germanas - grosso modo, ilustradas-, la que nos impediría ver, en el encuentro de estas dos frases, "Macedonio Fernández no vende" y "Roberto Bolaño sí vende", una contradicción que sólo se puede resolver por medio de un análisis de la entidad texto-autoral de Roberto Bolaño a la

5 "Mientras en Francia el concepto de civilización mantiene su definición corregida por el neoclasicismo de la Ilustración y, lejos de afirmarse en contradicción frente a la idea de cultura, pretende incluirla y definirla como la versión más refinada de sí misma, en Alemania el concepto de cultura se vuelve romántico, define a ésta como el resultado de la actividad del 'genio' creador y reduce la civilización a mero resultado de una actividad intelectualmente calificada" (Echeverría $30)$. 
luz de la teoría del valor de Marx, pues la primera, es decir, la trascendencia del campo literario nos mostraría que, en efecto, tras las condiciones establecidas como reglamentación al interior de éste es casi imposible que un autor como Macedonio Fernández "venda", mientras que la segunda, la aplicación endogámica de las herramientas filológicohermenéuticas nos mostraría, con un análisis (auto)de-limitado al corpus textual de Bolaño, que es imposible que una obra tal "no-venda". La respuesta a la pregunta por las condiciones de la nueva literatura latinoamericana sería, en este sentido, sólo posible por medio de una serie acumulativa de valores literarios (auto)impuestos a ciertas entidades texto-autorales en cuanto mercancías, o bien, lo que es lo mismo, sólo se podría formular en boca de alguien que "sí vende", y quien (hipotéticamente) explicara por qué los otros (Macedonio, Lamborghini, Wilock) no; es decir, si fuera posible, en efecto, formular la frase (hipotética) "Roberto Bolaño sí vende" ello sólo podría significar que ese nombre, Roberto Bolaño —el cual por otra parte, podría acaso coincidir con el de un autor y una serie de modalidades específicas de subjetivación por medio de la práctica editorial de Anagrama S.A. - sería completamente ajeno a la capacidad operativofenomenológica de la frase "Macedonio Fernández no vende", siendo, no obstante, el único capaz de enunciarla. Así pues, la frase (hipotética) "Roberto Bolaño sí vende" podría, por ejemplo, no sólo ser el título de la conferencia en Sevilla dictada por Roberto Bolaño, autor de Anagrama S.A. — con lo que, además de ser fieles a la verdad de los números se estarían reconociendo las capacidades actanciales del único escritor que puede decir el nombre del canon literario latinoamericano en cuanto a su centro se encuentra siempre diferido, (a)plazado 6 -, sino asimismo, implicaría una condicionante socio-cultural definitiva para la constitución de ulteriores modelos de seriación modal de prácticas

\footnotetext{
6 "En una entrevista el escritor chileno Carlos Franz relataba que, durante una charla de café, Bolaño se lamentaba de que la fama literaria le había llegado tardíamente. Franz sostenía que Bolaño estaba obsesionado con tener poder literario, ese poder que le otorga al escritor la posibilidad de delimitar un canon personal de adherencias y repulsas con una elevada dosis de arbitrariedad. ¿En su trato personal con Bolaño sintió esa obsesión que describe Franz? [...] Si lo tengo que deducir de mi trato personal con Bolaño, debía tratarse de una obsesión secreta. Quizá de una proyección de Carlos Franz. A Bolaño le gustaban los cánones (por definición, arbitrarios, cosa que no se le escapaba), las listas a lo Perec, pero como un juego. Un juego jugado razonablemente en serio, como debe ser" (Herralde, El optimismo 174).
} 
editoriales. En suma, si fuera posible decir - frente a una frase como la que enuncia Roberto Bolaño en la conferencia en Sevilla, esa, justamente, con la que hemos empezado a trazar la genealogía de las prácticas editoriales que, siendo producidas por el propio Bolaño en su condición de autor, lo producen, asimismo, a él一, o bien, mejor aún, si fuera posible escribir la frase (hipotética) "Roberto Bolaño sí vende" tendríamos ante nosotros el trazado de una relación dicotómica capaz de deconstruirse $-\mathrm{y}$, en esa deconstrucción, tendríamos asimismo la posibilidad de entender las condiciones de emergencia y las condiciones de posibilidad de la literatura latinoamericana finisecular y de inicios del siglo XXI, es decir, las posibilidades ampliadas de una genealogía de los procesos editoriales que estamos intentando rastrear y cuya lógica no podemos sino desprender de la práctica editorial a la que está subsumido (el autor) Roberto Bolaño. En suma, ¿qué implicaría decir "Roberto Bolaño sí vende" en el marco de una estrategia socio-cultural para la constitución de modelos de seriación modal de prácticas editoriales? Esta frase (hipotética), si bien para muchos puede ser, digamos, "cierta" en muchos sentidos, demuestra sencillamente que la otra frase - la que, en efecto, Bolaño enunció en Sevilla (y luego, escribió, o viceversa) - no tiene una contracara. La frase - sentencia, oración, mantra incluso- "Macedonio Fernández no vende", al desprenderse de su contexto de enunciación, no encuentra ninguna otra que se le resista porque ella misma es la resistencia absoluta a lo que entendemos, siguiendo a Bolívar Echeverría, como la dimensión cultural de la vida social, puesto que esta "puede intervenir de manera decisiva en la marcha misma de la historia. La actividad de la sociedad en su dimensión cultural, aun cuando no frene o promueva procesos históricos, aunque no les imponga una dirección u otra, es siempre, en todo caso, la que les imprime un sentido" (Echeverría 24). Me interesa subrayar que para el filósofo ecuatoriano esta dimensión cultural de la vida social se presenta como "un excedente o surplus ontológico que, en lugar de ser subsumido al tratamiento de las otras dimensiones de la reproducción social, debe ser tematizado de manera propia" (Echeverría 25), es decir, sin que se le subsuma al alcance (auto)de-limitado de la endogamia académico-mercantil regida por la utilización de la filología 


\section{AlFredo LÉAL RodRÍGUEZ}

y la hermenéutica como (seudo)herramientas de crítica literaria, por un lado, y fuera del alcance de las normas fenomenológicas del aparecimiento de dicha dimensión en el marco (de guerra) del campo literario. Dicho de otro modo, la única forma de entender la dimensión ontológica de la vida social en la que se encuentra de-tenida la contradicción entre los enunciados "Macedonio Fernández no vende" y "Roberto Bolaño sí vende" es si se la coloca, en cuanto surplus ontológico, en contraposición al excedente o plusvalor propio de la acumulación capitalista y de la valorización del valor, con lo cual entenderíamos la subsunción capitalista de la literatura en cuanto valor de uso, si lo hay, y en contra o en conflicto permanente con una dimensión no sólo civilizatoria sino también cultural de su propia existencia.

Ahora bien, si antes habíamos dicho que el enunciado "Macedonio Fernández no vende" — del mismo modo que ese otro (hipotético), "Roberto Bolaño sí vende" - es, principalmente, producto de una instancia nominal, a saber, posible gracias a que hay un sujeto de la enunciación capaz de decirlo, ¿quién hace empero posible esa enunciación, es decir, el acto de dar (el) nombre? En pocas palabras: ¿gracias a quién habla Bolaño (agregamos: de la forma precisa en la que habla)?

Hay que volver a Sevilla, entonces, pero no exactamente a la conferencia ni al texto que Bolaño escribe para después leer en ésta o bien dicta para después escribir sobre ésta. Se trata, creo, de volver a Sevilla sólo después de haber tratado de entender cómo se despliega la voz de Anagrama S.A. — pues ambas, Anagrama S.A. y Sevilla, conforman hasta ahora, en el decurso de esta exposición, un lugar editorial dúplice cuya contingencia y contradicción es preciso resolver, o intentar hacerlo, de manera dialéctica.

Qué hay, pues, de esa conferencia en Sevilla. Herralde menciona la conferencia en dos ocasiones en el transcurso del año 2003, la primera en el texto leído en el funeral laico de Bolaño ${ }^{7}$, donde la conferencia

${ }^{7}$ El texto "Adiós a Roberto Bolaño", "leído en el funeral laico del tanatorio Les Corts, Barcelona, 16 de julio de 2003", se publica, tal como está indicado en OE, en el diario La Vanguardia el 17 de julio de 2003 bajo el título "Bolaño, trapecista sin red". La imagen que acompaña la nota en La Vanguardia tiene el siguiente pie de foto: "Familiares y amigos del escritor —en primer plano, Jorge Herralde y Rodrigo Fresán - le dieron su último adiós en un funeral que congregó a un centenar de personas". 
forma parte de esos "indicios que permiten imaginar algo así como una despedida implícita" (Herralde, Para Roberto Bolaño 11), y la segunda en el homenaje que le realizan al autor en la Feria del Libro Chilena. ${ }^{8}$ En ambos documentos, de nuevo con ese efecto de naturalidad que ya hemos señalado como característico de sus textos, Herralde, si es que puede decirse utilizando la expresión de Borges, habla del Bolaño que "se dejaba vivir para que Bolaño pudiera tramar su literatura". Luego, la conferencia se menciona en dos entrevistas más, fechadas al año siguiente, en 2004, ambas asimismo contenidas en el volumen Para Roberto Bolaño. Sin embargo, queda claro que no es ese autor cuya vida le interesa —o bien, mejor dicho, no es ese autor el que está construyendo Herralde desde los textos anteriores a su muerte y (siempre) posteriores al Premio Herralde de Novela que le otorga en 1998. ${ }^{10}$ Pocos o nulos son los datos biográficos y proposopográficos

\footnotetext{
${ }^{8}$ Así comienza el texto de Herralde: "La muerte de Roberto Bolaño causó una extraordinaria conmoción en nuestro país, una explosión de pesar y de rabia con muy escasos precedentes. Muchos de los más destacados escritores y críticos literarios lo valoraron como el mejor escritor latinoamericano de su generación. Tan sólo unas pocas semanas antes, en una reunión de escritores latinoamericanos en Sevilla, la generación más joven, la de Fresán, Volpi o Gamboa, lo eligió como su líder indiscutible, su faro, su tótem, en palabras de Rodrigo Fresán. Y no sólo en España, en toda América Latina, en espacial en Chile y en México, se sucedieron cataratas de elogios y se expresó el dolor de la pérdida de un artista en su apogeo" (Herralde Para Roberto Bolaño 17). Congruente con su estilo, Herralde nombra una serie de autores y países que se van sumando al nombre de Roberto Bolaño; dicha serie, que va de lo general a lo particular, consigue un efecto hiperbólico: en unas cuantas líneas, Bolaño pasa de ser el "valorado como el mejor escritor latinoamericano de su generación" a convertirse en "el líder indiscutible, el faro, el tótem" de la que llama "la generación más joven” — en palabras, dice, del portavoz de la misma, Fresán—, para ser, finalmente, repatriado a América Latina, pues "no sólo en España” es donde se le reconoce como un artista que fallece "en su apogeo". La primera valoración de la que habla Herralde se deriva de textos y opiniones de críticos mayoritariamente españoles. Más adelante, en el mismo texto, luego definir a Bolaño como "un autor minoritario" (Herralde Para Roberto Bolaño 19), habla de la recepción que tuvo el chileno después de Los detectives salvajes, "publicado en noviembre del 98, que en pocos meses ganó nuestro premio de novela y el Rómulo Gallegos y de inmediato la unanimidad de los mejores críticos, como Ignacio Echevarría o Masoliver Ródenas, en España, Celina Manzoni en Argentina, Elvio Gandolfo en Uruguay, Christopher Domínguez-Michael, en México, o Rodrigo Pinto y Patricia Espinosa en Chile. Y también el instantáneo apoyo incondicional de escritores como Enrique Vila-Matas, Juan Villoro o, en Chile, Jorge Edwards, Jaime Collyer, Roberto Brodsky" (Herralde Para Roberto Bolaño 19-20).

${ }^{9}$ La frase de Borges proviene de su texto "Borges y yo", publicado en El hacedor (1960) y dice: "yo vivo, yo me dejo vivir para que Borges pueda tramar su literatura, y esa literatura me justifica” (Borges 808).

${ }^{10} \mathrm{El}$ autor-Bolaño que construye Herralde es un Bolaño que debe estar a la altura de las circunstancias que le exige el Premio que porta su nombre, porque la asignación de éste al chileno es un momento coyuntural: precisamente el primero de los textos que Herralde le dedica a Bolaño es, asimismo, la primera vez que el editor se expresa sobre el Premio Herralde de Novela por escrito:
} 
que Herralde comparte del autor chileno ${ }^{11} \mathrm{y}$, en su mayoría, los datos que da están relacionados con su escritura y lo que a Herralde, en tanto editor de Anagrama S.A., le corresponde. "Daba la impresión”, dice, por ejemplo, "de que Bolaño escribía como Kafka dijo, creo, que debía hacerse: escribir como si se estuviera muerto. Y esto me recuerda la forma cómo (sic.) Jacques Rigaut apostrofaba a sus amigos dadaístas menos radicales: "Vous êtes tous des poètes et moi je suis du côté de

"tal vez", dice, "como me han sugerido, sea este el momento de escribir un par de folios sobre el Premio Herralde" (Herralde, Opiniones mohicanas 109). El texto, datado el mismo mes de noviembre de 1998, es decir, a unos días de habérsele otorgado el Herralde de Novela a Bolaño - y aun cuando no se indica en ninguna de las dos ediciones de Opiniones mohicanas que ése sea el manuscrito que se leyó en la rueda de prensa del Premio Herralde 1998 - , se intitula precisamente "Roberto Bolaño, el otoño del premiado", en explícita referencia al título de la novela de García Márquez. "La hipótesis fundacional [del Premio Herralde], cuando se convocó en 1982", dice la voz de Anagrama S.A., "fue la posible existencia de la narrativa española aún ignorada después del páramo de los años setenta, marcados por el imperio de la Teoría, del Texto, del más escarpado Experimentalismo" (Herralde, Opiniones mohicanas 109. Las cursivas son mías). Después de hablar de los casos de Inglaterra, y el que él mismo denomina el "dream team", e Italia, con los emergenti, "posiblemente con más ruido que nueces", se pregunta "¿Por qué tan prometedores hallazgos no podían extrapolarse a la literatura española?" (Herralde, Opiniones mohicanas 110). La respuesta es iluminadora en muchos sentidos: "El resultado, feliz resultado, es que lo que se denominó 'nueva narrativa española' existía, aunque todavía agazapada, y que un buen número de sus componentes se han (sic.) ido presentando al Premio, a lo largo de estos años" (Herralde, Opiniones mohicanas 111). Hasta este momento, en un texto dedicado a Bolaño, el editor no ha hablado sino de literatura española. De este modo caracteriza a los ganadores: "La gran mayoría de los galardonados, por cierto, tiene un perfil similar: escritores excelentes, poco conocidos y más o menos (o muy) malditos comercialmente. Veneno para la taquilla, como decían en Hollywood" (Herralde, Opiniones mohicanas 111). Bolaño, dice Herralde, "coincide con tal perfil. Sus dos libros anteriores, Estrella distante y Llamadas telefónicas, pese a contar con reseñas excelentísimas de primeros espadas de la crítica - Ignacio Echevarría, Juan Antonio Masoliver Ródenas, Joaquín Marco, entre otros - vendieron poco más de unos centenares de ejemplares de cada título en España, y otros tantos en América Latina" (Herralde, Opiniones mohicanas 110).

Llama la atención que uno de ellos esté directamente relacionado con Sevilla: en la "Respuesta a un cuestionario de la revista 'Qué pasa' de Santiago de Chile", incluida asimismo en el libro Para Roberto Bolaño, Herralde relata su último encuentro con el autor. "El último día que vi a Roberto", dice, "me entregó el disquete de El gaucho insufrible y me comentó que finalmente [...] había desgajado 2666 en cinco novelas, cuatro terminadas y una quinta muy avanzada" (Herralde, Para Roberto Bolaño 65-66). Bolaño, a decir de Herralde, pasó un largo rato en las oficinas de Anagrama S.A., primero con Lali Gubern, luego con Teresa Ariño, encargadas de las traducciones y la edición, respectivamente, y al final, "como de costumbre, más de una hora" con Herralde. Bolaño hace "un repaso, bastante jocoso, a su estancia en Sevilla en ocasión de un comentado encuentro con escritores latinoamericanos algo más jóvenes que él, quienes lo consideraban su líder, aunque sus enfoques literarios fueran a menudo muy distintos (como documenta la polémica entre Ignacio Echevarría y Jorge Volpi)" (Herralde, Para Roberto Bolaño 66). Luego, al fin, el dato inesperado: "Me contó que uno de los motivos para el viaje a Sevilla fue la curiosidad por reencontrarse con una antigua novia a la que hacía muchos años que no veía. Un encuentro poco satisfactorio, por así decir, en torno al cual se explayó largamente" (Herralde, Para Roberto Bolaño 66-67). 
la mort ». Y a los muertos, si no otra cosa, la sinceridad se les supone" (Herralde Para Roberto Bolaño 28). Así pues, el autor-Bolaño que le interesa a Herralde es un autor que, con el nombre, ha recibido también una sentencia de muerte. Dar (el) nombre es dar (la) muerte. Y, en este sentido, el único modo de acceder a esa (com)pulsión es desplegando, a través, una vez más, de la serie de modalidades específicas de subjetivación, una "vida" que pueda, acaso, explicar esa muerte. ¡Feliz coincidencia, entonces, la muerte física de Bolaño, ese de quien "consta su perversa costumbre de falsear y magnificar", Borges dixit! La vida verdadera, para decirlo en términos de Proust, se ve confrontada a una vida otra, una vida que responde a las necesidades del proceso de acumulación de capital y, más allá, de valorización del valor. De este modo, la única vida posible para Bolaño desde la perspectiva de Herralde, desde la perspectiva de Anagrama S.A., es la vida anclada al lugar editorial, la vida editorial.

Llegamos así al que, creo, es el texto central de Jorge Herralde, recopilado en su libro Para Roberto Bolaño e intitulado precisamente "Vida editorial de Roberto Bolaño". "En el número 22, junio de 2004, de Vórtice", comienza Herralde, "Revista del Programa de Literatura Universidad del Mar, Antofagasta, se publicó un excelente reportaje de Felipe Ossandón titulado 'Reinventando a Bolaño. Ciudad de México y la aparición de su primer libro"' (Herralde, Para Roberto Bolaño 31). A partir de ese texto, es decir, de un acontecimiento editorial, Herralde traza una vida que co-incide con la-vida de Bolaño: cada uno de los aparecimientos del escritor están signados por un momento en su literatura. No es, stricto sensu, ni una biografía ni una prosopografía, sino un híbrido entre ambas mediado por la instancia específicamente literaria: "en 1976", dice Herralde, "además de aparecer junto con otros siete infrarrealistas en el libro colectivo Pájaro de calor, publicado por Ediciones Sánchez Sanchiz, edita su primer libro en solitario, el breve volumen de poemas Reinventar el amor, un largo poema dividido en nueve capítulos, en el Taller Martín Pescador que dirigía Juan Pascoe" (Herralde Para Roberto Bolaño 32). El autor sólo existe cuando ha publicado $\mathrm{y}$, de ese modo, se va perfilando un autor-Bolaño del que sólo importarán los datos valorizados. Así, luego del fracaso del 


\section{AlFredo LÉAL RodRÍGUEZ}

segundo libro en México y la antología Muchachos desnudos bajo el arcoíris de fuego (11 jóvenes poetas latinoamericanos), compilada por el propio Bolaño, "quien naturalmente es uno de los poetas, junto con otros dos infrarrealistas, Mario Santiago y Bruno Montané" (Herralde Para Roberto Bolaño 34), la narración se vuelca hacia lo que parece un aspecto biográfico, el nacimiento del hijo, Lautaro, el cual, empero, es leído por Herralde en estricta relación con su producción literaria: "Y sin dejar de cultivar la poesía decidió convertirse en novelista y así garantizar el futuro de su familia (una idea a priori pintoresca, pero finalmente acertada), y empieza a presentarse a toda clase de concursos literarios en las más diversas provincias españolas y cobrando de pasos algunos de esos 'premios búfalo', como los llamaba, indispensables para la supervivencia del piel roja" (Herralde Para Roberto Bolaño 34). Bolaño vive editorialmente, de acuerdo con lo que expone Herralde; su vida, la-vida de Bolaño, en este punto, deja de co-incidir con la vida editorial para que ésta la subsuma por completo. Podemos preguntarnos si es acaso esto lo que Bolaño hubiera escrito de sí mismo. En el mismo volumen, como anexo, se incluye una "Petición de la Beca Guggenheim" que data de noviembre de 1996, en la que Bolaño escribe:

Hasta los quince años viví en Chile. En 1968 mi familia se instaló en México. A los dieciséis años dejé los estudios y me puse a escribir y a trabajar (he ejercido todos los oficios). En agosto de 1973 volví a Chile, con tan mala fortuna que al cabo de un mes me tocó vivir el golpe de Estado. En enero de 1974 volví a México. Durante un tiempo participé en el Infrarrealismo, grupo poético vanguardista. En 1977 viajé a Europa y terminé instalándome en Barcelona. (Bolaño apud Herralde, Para Roberto Bolaño 78)

A diferencia de lo que se desprende del texto de Herralde, "escribir" y "trabajar", en el texto de Bolaño, no son una y la misma cosa; el énfasis que pone el autor en decir que "[ha] ejercido todos los oficios" - de manera, empero, un tanto humilde, entre paréntesis, apenas de pasoes, asimismo, fundamental, pues lo que en Herralde se convierte en una inmediata monetarización de la mercancía fuerza de trabajo por medio de los premios acá es más bien un listado, un recorrido vital. Es decir: mientras, para Herralde, los premios son simplemente momentos del 
proceso acumulativo bajo la forma equivalencial de la "supervivencia del piel roja", en Bolaño éstos siguen constituyendo, de algún modo, una riqueza: los premios de las provincias españolas no tienen ningún valor para Herralde más allá de los libros que rescata para Anagrama ${ }^{12}$; para Bolaño, en cambio $-\mathrm{y}$ acá tal vez sea pertinente mencionar que esta "Petición de la Beca Guggenheim" es, nada menos, para escribir Los detectives salvajes ${ }^{13}$ - , cada premio guarda un nombre, constituye una parte de la riqueza que ha ido acumulando y que sólo en ese proceso de obtención (posible) de la beca podría convertirse en capital. "Desde 1993", concluye Bolaño, "vivo única y exclusivamente de mi actividad como escritor profesional" (Bolaño apud Herralde, Para Roberto Bolaño 78). Para Herralde, "esos inicios editoriales en España" siguen siendo, empero, "casi clandestinos", hasta que "empieza una nueva etapa con La literatura nazi en América, que publicó Seix Barral [...] con muy escasas ventas, por lo que al cabo de un tiempo la edición fue guillotinada casi en su totalidad, lo que, como es lógico, resultó muy traumático para Bolaño, un episodio que no olvidó" (Herralde Para Roberto Bolaño 35). La literatura nazi en América es rechazada por Alfaguara, Destino, Plaza Janés, queda finalista del Herralde de Novela en 1995 y Bolaño decide retirarla del premio antes del fallo. "Me tomó totalmente por sorpresa", dice Herralde, "ya que, fuera el que fuera el resultado de nuestro concurso, pensaba publicarla y creía

12 "Entre los libros que recogieron trofeos figuran, sin pretensiones de exhaustividad, La pista de hielo, Premio Ciudad Alcalá de Henares, publicado por la Fundación Colegio del rey en 1993, luego publicado por Seix Barral en Chile y años después en España; La senda de los elefantes, Premio de Novela Corta Félix Urabayen, publicada por el Ayuntamiento de Toledo en 1993, luego editada por Anagrama con el título Monsieur Pain; Los perros románticos, Premio Ciudad de Irún de Poesía, publicado por la Sociedad Guipuizcoana. A esos títulos debe añadirse la novela, escrita con Antonio García Porta, Consejos de un discípulo de Morrison a un fanático de Joyce, que gano el Premio Anthropos" (Herralde, Para Roberto Bolaño 35). Todos los premios que destaca Herralde datan precisamente de 1993; Bolaño incluye en la "Petición de una Beca Guggenheim" uno más, anterior a ese año: "el Ámbito Literario de Narrativa, en 1984" (Herralde, Para Roberto Bolaño 78).

13 "La petición estaba acompañada de una carta mía", dice Herralde, "como editor suyo, una fotocopia de una carta de Silvia Meucci, scout de Fertrinelli, comunicándole su informe positivo de Estrella distante, aconsejando su publicación (recomendación desoída por la editorial italiana), así como de las reseñas de Estrella distante a cargo de Joaquín Marco $(A B C)$, Ignacio Ecvhevarría (El País) y Luis Alonso Girgado (El correo Gallego) [...] El 2 de mayo de 1997, en una carta de la John Guggenheim Memorial Foundation, Peter Kardon, director del Latin American Program, deploraba comunicar que, a causa de los limitados fondos de subvención de dicho año, el Comité de Selección no había podido subvencionar a Roberto Bolaño" (Herralde, Para Roberto Bolaño 77). 


\section{Alfredo LÉAl RodrígueZ}

(ingenuamente, no conocía aún sus artimañas de supervivencia) que nos la había enviado sólo a nosotros" (Herralde, Para Roberto Bolaño 36). Ese es el episodio crucial, el que propicia el encuentro.

Le escribí diciéndole que si algún día venía a Barcelona, me gustaría hablar con él, y añadí, un tanto escuetamente (quizá algo cabreado, los amores despechados), que La literatura nazi en América 'me causó buena impresión, aunque dudaría de calificarla como novela'. Me llamó a los pocos días, pasó por Anagrama, estuvimos charlando largo rato, me habló de sus penurias económicas y de su desesperación por los muchos rechazos editoriales, por lo que cuando llegó una carta de Mario Lacruz con una oferta, lógicamente módica, por La literatura nazi, no lo dudó un segundo. Y, hablando de rechazos, me recordó que la novela que escribió con Antonio García Porta también la había presentado en su día a nuestro premio y quedó preseleccionada en la lista larga de diez finalistas, pero no prosperó. Le dije que, si no tenía compromiso con otras editoriales, me encantaría leer otros textos suyos y al poco tiempo me trajo Estrella distante (luego me enteré de que también había sido rechazada por otras editoriales, incluso por la propia Seix Barral), que me pareció extraordinaria, y así empezó nuestra relación editorial y amistosa. Se publicó unos meses después que La literatura nazi, en otoño de 1996, y la primera rueda de prensa en la vida de Bolaño se celebró el 25 de noviembre de 1996. (Herralde, Para Roberto Bolaño 36-37. Las cursivas son mías)

A pesar del éxito entre los críticos (Herralde Para Roberto Bolaño 37-40), el primer libro de Roberto Bolaño autor de Anagrama S.A. no logra las ventas esperadas. "Como ejemplo", dice Herralde, "se vendieron en todo el mundo, incluida América Latina, 951 ejemplares de Estrella distante durante el primer año, 816 el segundo y 818 el tercero" (Herralde, Para Roberto Bolaño 40). Las ventas de Bolaño empiezan a ser mayores a los mil ejemplares sólo a partir de Llamadas telefónicas, que llega a 2,651 ejemplares en 1997 y 849 en 1998 (Herralde, Para Roberto Bolaño 41). "Sin embargo", dice el editor, "la explosión cualitativa y cuantitativa no se produjo [sino] hasta Los detectives salvajes, en 1998, que inaugura una tercera etapa en la vida editorial del autor" (Herralde, Para Roberto Bolaño 41. Las cursivas son mías). 
¿Cómo podemos entender esas tres etapas de las que ha hablado Herralde — con base solamente en datos editoriales? Me parece que, en un primer momento, estas tres etapas corresponden a una especie de desarrollo vital editorial — que, por cierto, podría aplicarse a todo autor $\mathrm{y}$ autora con base justamente en la relación que mantiene el proceso de valorización de sus correspondientes entidades texto-autorales-: 1) la infancia editorial de Bolaño en México, después del primer exilio, determinada por su participación en el infrarrealismo y los respectivos libros de poesía; 2) la adolescencia editorial de Bolaño, sus "artimañas de supervivencia", los primeros libros españoles, los rechazos, la desesperación por los mismos y el paso a la juventud editorial, que correspondería con los primeros dos libros-fetiche ${ }^{14}$ en Anagrama; 3 ) la adultez, posterior al Premio Herralde de Novela, con Los detectives salvajes: "Se comparó Los detectives salvajes con las mejores novelas latinoamericanas del siglo XX, como Rayuela o Adán Buenosayres, y para muchos de los jóvenes escritores de las nuevas generaciones se convirtió en un ejemplo, un modelo, un héroe, destronando, o desplazando, o aparcando a muchas figuras señeras del boom" (Herralde, Para Roberto Bolaño 42). Habría, finalmente, una última etapa, 4) la vejez literaria, "la última etapa de esta crónica de la vida editorial de Bolaño: la difusión internacional de su obra, sus traducciones, cuyo número no cesa de aumentar, la extraordinaria acogida crítica que ha tenido, la fidelidad de sus editores, que a menudo han sido dos por país, a causa de sus numerosos títulos, como también le sucede a Vila-Matas" (Herralde, Para Roberto Bolaño 45). Esta etapa final de la vida estaría, empero, seguida de una especie de posvida, un más allá que, en el caso de Bolaño, está cifrado por la novela póstuma, con la que concluye

\footnotetext{
14 "Yo mismo conocí bien los fetichismos editoriales de Roberto: uno era publicar al menos un libro al año en Anagrama, y así sucedió. También escrutaba minuciosamente nuestros catálogos y boletines, en especial Deconstructing Anagrama, que habíamos publicado en ocasión de los 30 años, en 1999. En éste figura, a modo de prólogo, una lista de autores con 10 o más títulos en el catálogo de Anagrama, el 'club de los 10', como lo llamábamos. Un club compuesto por 18 escritores en 1999 y al que quería acceder lo antes posible y codearse con sus admirados Pombo y Vila-Matas. Ahora [2004-2005], en el Deconstruting Anagrama de los 35 años, Bolaño, con 11 títulos, ya figura en el club (que se ha ampliado hasta 27 escritores)" (Herralde, Para Roberto Bolaño 33-34). En una conferencia en Princeton en 2008, Herralde dice: "tuve la oportunidad de reconocer a Bolaño. Y el posible mérito fue haber armado un dispositivo, es decir, un catálogo, con autores tan atractivos como para que un autor literariamente muy ambicioso como Roberto Bolaño quisiera formar parte del club" (Herralde, El optimismo de la voluntad 293).
} 
Herralde su "Vida editorial de Roberto Bolaño": "Y me detengo aquí, al borde de 2666, recién publicada esta misma semana, que leí enfebrecido en manuscrito hace más de un año y cuya edición ha ido a cargo de Ignacio Echevarría. Según los pocos lectores que la han leído, tanto aquí como todavía en pruebas algunos colegas internacionales, se trata de una novela tan importante que puede afirmarse, sin ninguna duda y sin rehuir los tópicos, que es el primer gran clásico del siglo XXI" (Herralde Para Roberto Bolaño 47). No obstante, estas etapas, así identificadas, no explican sino de manera superficial la vida de Bolaño subsumida a la edición, precisamente porque esa subsunción entendida bajo la categoría de "vida editorial" — si bien algunas entidades textoautorales de Bolaño dan cuenta de ésta, como lo son, por ejemplo, el cuento "Sensini", en el que relata el proceso de supervivencia con los premios de provincia; el propio Arturo Belano en tanto representación sígnica de Bolaño; o bien la relación con México y las llamadas muertas de Juárez mediante Sergio González Rodríguez ${ }^{15}$ — es mucho menos una mercantificación de la vida que una antropoformización de la obra.

Este ciclo vital, signado por la pulsión de muerte, se convierte así en un ciclo que reproduce los momentos de producción, circulación y consunción de las mercancías. Aquí, empero, no se trata del modo singular en el que cada una de las entidades texto-autorales de Bolaño se produce, circula y consume, sino cómo cada una de éstas — dependiendo del momento en el que se ubican — producen, hacen circular y consumen la mercancía a la que están sujetas. Dicho de otro modo: de lo que se trata, en los textos de Herralde, en la que hemos denominado su práctica editorial, es de con-formar la mercancía-Bolaño de tal modo que sea posible ubicarla en diversos momentos vitales: las mercancías sólo viven en el ciclo constituido por la producción, la circulación, la consunción y la generación de plusvalor, y la mercancía-Bolaño se conforma a partir de una evolución, un currículum vitae de la mercancía desde su aparecimiento monádico hasta su espectralización en la forma de un capitalismo financiero. Los momentos de producción, circulación,

\footnotetext{
15 "En la novela [2666] aparece un joven reportero que empieza a investigar. Se trata de Sergio González Rodríguez, que al final de la novela toma el relevo de las pesquisas más avanzadas. Roberto y Sergio estuvieron en contacto frecuente respecto a las muertes en Ciudad Juárez, por e-mail y con una visita de Sergio a Blanes" (Herralde Para Roberto Bolaño 64).
} 
consunción y generación de plusvalor se identifican mediante los esquemas básicos del intercambio mercantil, a saber:

a) en la infancia (Bolaño-poeta en México), el intercambio simple (M $-\mathrm{M})$

b) en la adolescencia (Bolaño-Sensini narrador en España), el intercambio mercantil dinerario $(\mathrm{M}-\mathrm{D}-\mathrm{M})$;

c) en la adultez (Bolaño-Belano autor de Anagrama y ganador del Premio Herralde), el intercambio capitalista propiamente dicho (D $-\mathrm{M}-\mathrm{D})$; $\mathrm{y}$, finalmente

d) en la vejez/muerte (Bolaño-transnacional, artífice de 2660), el intercambio financiero por excelencia (D - D), en donde la folía del capital trastorna el propio corpus textual - imagen, desdoblamiento y diseminación del cuerpo del autor - en momentos de trascendencia desmesurada.

Podríamos representar gráficamente esta vida editorial de la siguiente forma:

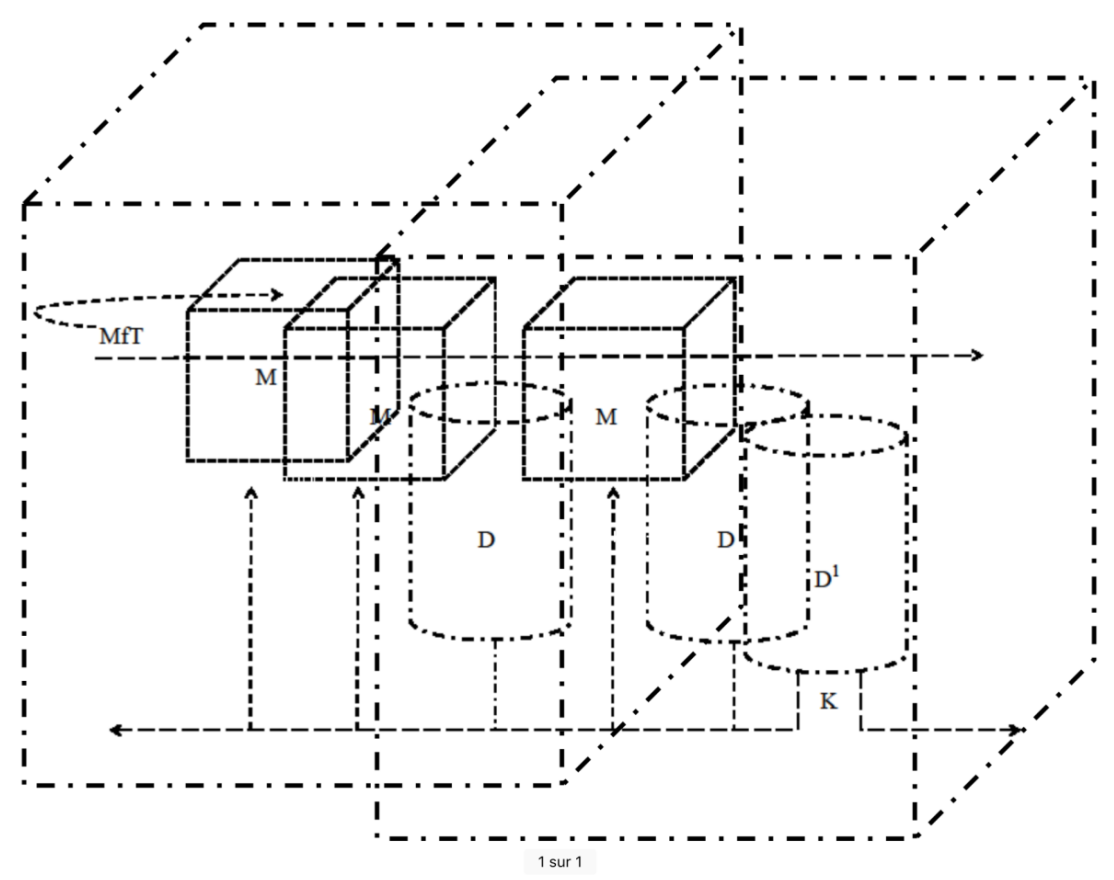


La mercancía-Bolaño es, como todas las mercancías-autor, bifacetica. ${ }^{16}$ En este caso, del encuentro de la biografía y la prosopografía se produce un espacio que podemos denominar como instancia material, y es al interior de ésta, contaminando ambas, biografía y prosopografía, que se encuentra la ontología de la con-formación de la mercancíaBolaño, entendida en el sentido de una red o sistema de datos que define las relaciones existentes entre las mercancías (libros) que constituyen esa mercancía en un nivel superior. Dicho de otro modo, la vida de la mercancía-Bolaño es su vida editorial entendida como la relación que mantienen sus libros entre sí y en un sentido siempre acumulativo en busca de la generación del plusvalor. Así pues, al interior de la instancia material tendríamos la mercancía fuerza de trabajo (MfT) vaciándose en una primera mercancía, por un lado, y recorriendo de manera horizontal todas las demás; este proceso de vaciamiento es fundamental para entender la vida editorial, en el sentido en el que es el origen mismo de una obra, entendida en los términos de la filología y la hermenéutica,

\footnotetext{
${ }^{16}$ Para Bolívar Echeverría, el proceso de producción/consumo de objetos (prácticos) tiene un correlato en el proceso de producción/consumo de significaciones. Este paralelo, empero, "afirma que entre el proceso de producción/consumo de objetos prácticos y el proceso de producción/ consumo de significaciones hay una identidad esencial" (Echeverría 85). Esa es la base, afirma Bolívar, para la definición de la cultura, de la cual se desprenden dos tipos de objetos: "los primeros serían aquellos objetos que presentan menos forma en más material, aquellos cuya materialidad es menos dúctil" (Echeverría 86). De este tipo de objetos, Bolívar pone como ejemplo una casa, debido a que ésta tiene una agilidad baja para transmitir un sentido comunicativo —o bien, agregamos nosotros, debido a que el sentido comunicativo que transmite es unívoco, a saber, limitado a una significación programática. El segundo tipo de objetos, empero, "a la inversa, serían objetos que en menos material tienen más forma, aquellos cuyo material es más sutil, más apto para asumir formas y cuyo tiempo de producción/consumo es más corto" (Echeverría 86). De éstos, dice Bolívar, el ejemplo sería un guiño de ojos que transmite un sentido comunicativo a través de una capacidad relativamente alta o más ágil, que, agregamos, puede identificarse con la transmisión de un sentido comunicativo equívoco; a saber, mientras una casa, una vez que se ha identificado como tal en el momento de producción, sólo puede ser una casa, un guiño de ojos puede ser muchas otras cosas. El libro en cuanto instancia material que funciona como momento equivalencial entre biografía y prosopografía debe entenderse como la síntesis entre ambos tipos de objetos prácticos: en cuanto material que excede la forma, el libro ("en acto") transmitiría un sentido inmediato aunque de manera menos ágil, y sería el soporte de un mensaje; en cuanto forma que excede el material, el libro ("en potencia") transmitiría un sentido mediado siempre por la lectura en cuanto praxis, más ágil, sí, pero más lentamente también, y sería el portador de un mensaje. La instancia material se con-formaría, de este modo, como un corpus textual adánico y no paulino, es decir, como el cuerpo que significa en tanto cuerpo $y$ alma y no en cuanto cuerpo $o$ alma. Creemos que el propio Bolívar intuía la importancia que tiene el libro en cuanto instancia material para su teoría cuando dice que esos objetos cuya forma excede la materia "llegan a tener el máximo de

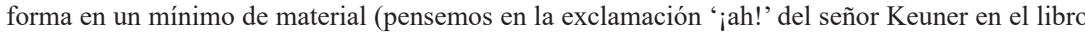
de Brecht)" (Echeverría 87).
} 
o bien de un autor, entendido en el sentido del campo literario. La primera mercancía (M) al extremo izquierdo de la gráfica se encuentra de manera equivalencial con otra $(\mathrm{M}-\mathrm{M})$ sin que entre ellas haya una mediación dineraria, la cual no surge sino hasta el encuentro de la segunda mercancía con una tercera, mediado, en el caso de Bolaño, por el premio de provincia (D); así, entre las mercancías dos y tres se genera un intercambio explícitamente dinerario $(\mathrm{M}-\mathrm{D}-\mathrm{M})$ que constituye la mercancía cuatro. Este intercambio no es aún capitalista, pues éste vendrá hasta el momento en el que la mercancía cinco, penúltima en la gráfica, se coloque en el extremo correspondiente al valor de uso (D - M - D); en este punto, para decirlo brevemente, el libro de Bolaño es, sobre todo, dinero que generará más dinero; esta mercancía es precisamente Los detectives salvajes, producida por el Premio Herralde y productora, entre otros, del Rómulo Gallegos, y a la cual le seguirá, entonces, el verdadero momento de generación del plusvalor, el excedente al que está sometida la vida editorial de Bolaño contrapuesta a una formación vital que admita la dimensión cultural de la vida social sin que ésta se subsuma a la instancia capitalista. Así, la mercancía final al extremo derecho de la gráfica $\left(\mathrm{D}^{1}\right)$ genera capital $(\mathrm{K})$ en dos sentidos: el primero, prospectivo (hacia la derecha de la gráfica), posibilita, entre otras cosas, las traducciones de Bolaño, aunque también es el que produce las demandas que Carolina López ha impuesto contra Carmen Pérez de Vega o Ignacio Echevarría, los coloquios internacionales sobre la obra de Bolaño o el parque de diversiones temático que en su honor se ha realizado en la China; el segundo sentido, retrospectivo, vacía ese capital sobre las mercancías que, en el flujo vital-editorial, se han ido acumulando, lo cual permite no sólo asignarle un valor de cambio a los primeros libros y las primeras ediciones; esto explica, entre otras cosas, por qué esas primeras ediciones cuestan lo que cuestan hoy día, siendo que, en su momento, no sólo no tenían un equivalente dinerario sino que se intercambiaban por otras mercancías - libro por libro.

Las mercancías representadas por cubos (M) difieren, pues, de aquéllas que hemos identificado con cilindros (D) en cuanto a que estas últimas pertenecen, desde su formación, a la lógica de una economía capitalista, mientras que las primeras son, digámoslo así, la primera 


\section{Alfredo LÉAl RodrígueZ}

aproximación a ésta. El caso de Bolaño es central para nuestro estudio de la edición en cuanto serie de modalidades específicas de subjetivación de entidades texto-autorales debido a que en éste se cumple el proceso vital-editorial de la manera más simple y perfecta posible, lo cual no quiere decir, empero, que este esquema no se reproduzca en el caso de otras entidades texto-autorales. Podemos decir, sin temor a equivocarnos, que una entidad texto-autoral es tal sólo en la medida en la que se encuentra ubicada en un lugar editorial localizable en eso que hemos llamado instancia material con base en el caso de Bolaño, es decir, una entidad texto-autoral es tal si y sólo si es una mercancía cuyo interior está conformado por esta vida editorial entendida como un proceso de acumulación que concluye con la generación de plusvalor. Lo que parece radicalmente diferente en el caso de Bolaño con respecto a las mercancías (entidades texto-autorales) del Boom - por no hablar de casos más lejanos, como el martinfierrismo en Argentina o los propios estridentistas en México, referencia obligada para la con-formación de la mercancía Bolaño, y mucho menos de los casos anclados aún a un momento anterior a la modernización de la literatura, a saber, de la novela panfletaria hacia atrás, cuya producción estaba basada, sobre todo, en la relación que la literatura mantenía con los diversos desarrollos urbanos, como se ve no sólo en la obra de Martí o Darío sino en la del propio Balzac - es que en ésta el editor juega un lugar central no sólo como poseedor de los medios de producción sino, tal como lo hemos venido indicando a lo largo de este artículo, como autor de autores. Es por ello que el presente esquema, de ser factible, representaría un momento coyuntural en la constitución de la literatura latinoamericana. En suma, esta con-formación de la vida de Bolaño en torno a su vida editorial constituye un primer y decisivo paso para el paradigma de la producción, circulación y consunción de autores en Latinoamérica, paradigma que, después del Boom, toma a la mercancía-Bolaño como referencia fundacional y unívoca. Así pues, quien es capaz de enunciar la frase "Roberto Bolaño sí vende" es exactamente la misma entidad texto-autoral que enuncia la frase, otra, que hemos utilizado como enclave para trazar las coordenadas de una genealogía de la práctica serial de edición finisecular y de principios 
del siglo XXI que hemos identificado bajo el nombre (provisional) de editorialitarismo, es decir, "Macedonio Fernández no vende": la entidad texto-autoral que se desprende no la de (1 autor) Bolaño sino la entidad texto-autoral cuyo correlato es la mercancía-Bolaño.

Quien es capaz de enunciar las condiciones de la Literatura Latinoamericana es el poseedor de las mercancías-autor, en este caso, el poseedor de la mercancía-Bolaño.

Quien es capaz de enunciar es el poseedor de las mercancías-autor.

Quien es capaz de enunciar es el poseedor de las mercancías.

Quien es capaz de enunciar es el editor.

\section{Referencias}

Bolaño, Roberto. "Sevilla me mata". El secreto del mal. Barcelona: Anagrama, 2007.Impreso.

Borges, Jorge Luis. "Borges y yo". Obras completas 1923-1972. Buenos Aires: Emecé, 1974. Impreso.

Echeverría, Bolívar. "La dimensión cultural de la vida social". Definición de la cultura. México: FCE, 2010. Impreso.

Herralde, Jorge. Opiniones mohicanas. Barcelona: Acantilado, 2001. Impreso. Herralde, Jorge. Para Roberto Bolaño. Barcelona: Acantilado, 2005. Impreso. Herralde, Jorge. El optimismo de la voluntad. México: FCE, 2009. Impreso. Rancière, Jacques. "Le sociologue roi". Le philosophe et ses pauvres. Paris: Flammarion (Col. Champs), 2007. Impreso.

Ricœur, Paul. "Une phénoménologie de l'homme capable". Parcours de la reconnaissance: Trois études. Paris: Gallimard (Col. Folio Essais), 2009. Impreso. 